\title{
miR-27a promotes proliferation, migration, and invasion of colorectal cancer by targeting FAM172A and acts as a diagnostic and prognostic biomarker
}

\author{
WENJUN LIU ${ }^{1 *}$, KAI QIAN $^{1 *}$, XING WEI $^{2}$, HAIJUN DENG ${ }^{3}$, \\ BEI ZHAO $^{1}$, QING CHEN ${ }^{1}$, JINQIAN ZHANG ${ }^{4}$ and HAO LIU ${ }^{1}$ \\ ${ }^{1}$ Department of Vascular Surgery, Nanfang Hospital, Southern Medical University; \\ ${ }^{2}$ The First Clinical Medical College, Southern Medical University; ${ }^{3}$ Department of General Surgery, \\ Nanfang Hospital, Southern Medical University, Guangzhou, Guangdong 510515; \\ ${ }^{4}$ Department of Laboratory Medicine, The Second People's Hospital of Guangdong Province, \\ Guangzhou, Guangdong 510317, P.R. China
}

Received October 21, 2016; Accepted April 10, 2017

DOI: $10.3892 /$ or.2017.5592

\begin{abstract}
Accumulating evidence shows that mircroRNAs (miRNAs) play a crucial role in the development of colorectal cancer. In our previous study, FAM172A was demonstrated to be a novel tumor suppressor gene in CRC. Therefore, the aim of the present study was to identify whether the miR-27a could be a diagnostic and prognostic marker and the regulatory relationships between miR-27a and FAM172A. We demonstrated high levels of miR-27a expression in tissues of patients with CRC as well as in CRC cell lines. There was a positive correlation between the levels of miR-27a and the poor overall survival of patients with CRC. Furthermore, elevated levels of miR-27a expression were associated with TNM stage and distant metastasis. Increased expression or inhibition of miR-27a promoted or inhibited the metastasis of CRC cell lines, respectively. Moreover, we showed that miR-27a directly targets the 3'-untranslated region of FAM172A mRNA by using a dual-luciferase assay. Increased or decreased expression of FAM172A expression was observed when miR-27a expression was inhibited or elevated in the CRC cells, respectively. In summary, our study showed
\end{abstract}

Correspondence to: Dr Hao Liu, Department of Vascular Surgery, Nanfang Hospital, Southern Medical University, Guangzhou, Guangdong 510515, P.R. China

E-mail: droctorliu24@163.com

Dr Jinqian Zhang, Department of Laboratory Medicine, The Second People's Hospital of Guangdong Province, Guangzhou, Guangdong 510317, P.R. China

E-mail: sciencejqzhang@sina.com

*Contributed equally

Key words: miR-27a, colorectal cancer, metastasis, FAM172A, diagnosis, prognosis that miR-27a expression is a diagnostic and prognostic marker and correlates with overall survival of patients with CRC. Therefore, it may be a therapeutic approach for preventing metastasis of CRC to inhibit expression of miR-27a or increase expression of FAM172A.

\section{Introduction}

Colorectal cancer (CRC) is one of the most common cancers worldwide because of its high metastasis rate, and lack of widely accepted prognostic marker (1). Cancer metastasis is a complicated process involving the cell ability to initiate and maintain growth at a distant site and increase morbility (2). Unfortunately, although diagnosis and treatment of CRC have made great progress, available measures to predict or prevent metastasis of CRC are absent, which leads to the poor clinical outcome and prognosis of CRC patients with metastasis who usually have genetic and epigenetic abnormalities, such as KRAS mutation and microsatellite instability (3-7).

In recent years, we have focused on studying the mechanisms of the novel gene FAM172A, the family with sequence similarity 172 . This functionally unknown gene was first identified at the translational level through western blotting (8), and it was regulated by high glucose levels in human THP-1-derived macrophages (9). Our previous study showed that FAM172A expression in cancerous tissues was obviously lower compared with adjacent tissues of CRC cells by using immunohistochemical staining (10). In addition, our other past results showed that FAM172A could promote apoptosis and inhibit proliferation of CRC cell lines by flow cytometry and STAT1 could upregulate the expression of FAM172A when it was combined with the promoter region of FAM172A, indicating that FAM172A might be a novel tumor-suppressor gene (11). However, more molecular mechanisms of FAM172A underlying this process are still to be elucidated.

The structure and functions of FAM172A was analyzed recently by us using bioinformatics method and it was found that FAM172A is a target of a variety of miRNAs, such as 
miR-27a, miR-26b and miR-135. As small non-coding RNA molecules, microRNAs (miRNAs) can affect extensive biological processes endogenously (12-14). miRNAs can bind to the 3'-untranslated region (UTR) of targeting messenger RNA (mRNA), thus regulating protein-coding gene expression by repressing translation (15-18). There is plenty of evidence that miRNAs are an important influence on human oncogenesis and metastasis $(19,20)$ by regulating the expression of a variety of pivotal genes, such as miR-9, miR-153 and miR-145 (21-27). Approximately 30-60\% of the protein-coding human genome genes are regulated by miRNAs (28). In consequence, to identify tumor-related miRNAs and their mediated novel cancer networks is a key step to illustrate the molecular mechanisms of development of human CRC. Among numerous miRNAs, miR-27a has attracted our research interest. Recent reports have shown that miR-27a represses the expression of MHC class I by downregulating calreticulin thus affecting tumor progression in colorectal cancer, which was considered an oncomiRNA (29). Zhou et al confirmed that BTG2 was a direct target of miR-27a in gastric cancer and miR-27a inhibition obviously upregulated the expression of BTG2 (30). In light of these findings, we propose the hypothesis that miR-27a functions as an oncomiRNA and plays an important role in the development of CRC, especially in the progress of CRC invasion and metastasis by regulating FAM172A.

To test or hypothesis, we detected the expression of miR-27a in CRC samples. We uncovered that miR-27a was upregulated in colorectal cancer cell lines and tissues and that may act as an important prognostic factor for overall survival (OS) of patients with CRC. Then we studied the effects of miR-27a in proliferation, migration and invasion of CRC cells. In the present study, we showed that FAM172A was a direct target of miR-27a by using a dual-luciferase assay and the expression of FAM172A was regulated by miR-27a in CRC cell lines by using real-time PCR and western blotting. These results demonstrated that miR-27a promotes metastasis of CRC cell lines by inhibiting FAM172A expression, which provides a better understanding of how miR-27a regulates mechanisms of FAM172A in the development of CRC.

\section{Materials and methods}

Patient tissue samples. A total of 60 pairs of CRC and adjacent normal colorectal tissue (ANCT) samples were obtained from patients who underwent surgery between January 2010 and December 2015 at Nanfang Hospital, Southern Medical University (Guangzhou, China). The tissue specimens were stored in liquid nitrogen. None of the patients had received chemotherapy or radiotherapy before the surgery. Informed consent was obtained from all study participants. The study protocol was approved by the Ethics Committee of Nanfang Hospital, Southern Medical University, Guangzhou, China.

Cell cultures. Human colorectal cancer cell lines (LoVo, HT-29, SW480, HCT116, DLD-1 and RKO) and normal human colonic epithelia (NCM460) were purchased from the Type Culture Collection of the Chinese Academy of Sciences (Shanghai, China). The cell lines were cultured in RPMI-1640 (Hyclone, USA) supplemented with $10 \%$ fetal bovine serum (FBS) and were incubated at $37^{\circ} \mathrm{C}$ with $5 \% \mathrm{CO}_{2}$.
Quantitative real-time $R T-P C R(R T-q P C R)$. Total RNA was extracted using TRIzol (Beyotime, China) from the cultured cells, and total miRNA was extracted using RNAiso for small RNA (Takara, Japan) from the cultured cells and human tissues according to the manufacturer's instructions. For analyzing mRNA expression of genes, reverse transcription was performed using the First Strand cDNA Synthesis ReverTra Ace kit (Toyobo, Japan), and the SYBR Green qPCR Master Mix (Takara) was used carring out qPCR with the ABI 7500 Real-Time PCR system according to the manufacturer's instructions. For miRNA analysis, real-time PCR was performed using PrimeScript ${ }^{\circledR}$ miRNA RT-PCR kit (Takara) according to the manufacturer's instructions. The PCR conditions used were $95^{\circ} \mathrm{C}$ for $30 \mathrm{sec}$, followed by 40 cycles of $95^{\circ} \mathrm{C}$ for $5 \mathrm{sec}$, and $60^{\circ} \mathrm{C}$ for $30 \mathrm{sec}$. U6 snRNA and GAPDH were used for normalization for miRNA and mRNA, respectively. The relative expression was analyzed by the $2^{-\Delta \Delta C t}$ method (31). The sequences of the miRNAs and mRNA used in the present study are shown in Table I.

Bioinformatics analysis. TargetScan (http://www.targetscan. org/) and microRNA.org (http://www.microrna.org/microrna/) algorithm were used for identifying the potential targets of miR-27a.

Western blotting. The RIPA buffer was used in lysing cells. The Bradford protein assay (Bio-Rad, CA, USA) was used in quantifying total protein. Protein samples were denatured at $100^{\circ} \mathrm{C}$ for $5 \mathrm{~min}$. For electrophoresis in $10 \%$ sodium dodecyl sulfate-polyacrylamide gels, $30 \mu \mathrm{g}$ of total protein were loaded equally and then transferred to polyvinylidene fluoride microporous membranes (Millipore, MA, USA). Then samples were incubated with primary antibody for $12 \mathrm{~h}$ at $4^{\circ} \mathrm{C}$ which were followed by incubation with secondary antibody for $1 \mathrm{~h}$ at room temperature. Then, target proteins were detected with enhanced chemiluminescence reagents (Millipore). The primary antibodies including anti-FAM172A (1:700 dilution) (ab121364), anti-GAPDH (1:3,000 dilution) (ab8245), anti-MMP-2 (1:1,000 dilution) (ab25483), anti-MMP-9 (1:1,000 dilution) (ab76003), anti-NF-кB (1:1,500 dilution) (ab32536) and HRP-conjugated goat anti-rabbit igG (1:5,000 dilution) (ab6721) were from Abcam Group (Abcam, UK).

Dual-luciferase reporter assay. The genome DNA was derived from HEK293T cells, and then the 3'-untranslated region (UTR) of FAM172A was amplified by PCR, which contained the putative miR-27a target sites and the synthetic mutant 3'-UTR of FAM172A. Then products from PCR were cloned into psiCHECK-2 vector (Promega, USA). All inserts were sequenced to verify polymerase fidelity after digested by XhoI and NotI. Subsequently HEK293T cells were cultured in 24-well plates. The $50 \mathrm{nM}$ of miRNA mimic and $200 \mathrm{ng}$ of psiCHECK-2 vector containing 3'-UTR of FAM172A were cotransfected into each well of 24-well plates using Lipofectamine $^{\circledR} 3000$ (Invitrogen, USA). After $48 \mathrm{~h}$, the analysis of results was performed using the dual-luciferase reporter assay system (Promega) accordance with the protocol. Firefly luciferase activity was used normalizing Renilla luciferase activity. Experiments were carried out at least in triplicate. 
Table I. Sequences of RNA or DNA oligonucleotides.

\begin{tabular}{lll}
\hline Name & \multicolumn{1}{c}{ Sense strand/sense primer (5'-3') } & Antisense strand/antisense primer (5'-3') \\
\hline $\begin{array}{l}\text { Primers for RT-PCR } \\
\text { miR-27a }\end{array}$ & CTAATCGTGTTCACAGTGGCTAAG & TATGGTTTTGACGACTGTGTGAT \\
FAM172A & CAACGAGAAGCCGATGTA & GATGTGTCTAATGGTTCTGAG \\
MMP-2 & GGGAGATCATCGGGACAACTC & GGGCCTGGTTGAAAAGCAT \\
MMP-9 & CCTGGAGACCTGAGAACCAATC & CCACCCGAGTGTAACCATAGC \\
NF- $\mathrm{kB}$ & AGCACGACAACATCTCATT & GGCACAACTCCTTCATCC \\
GAPDH & ACCCACTCCTCCACCTTTG & CACCACCCTGTTGCTGTAG
\end{tabular}

Table II. Sequences of RNA or DNA oligonucleotides.

\begin{tabular}{llc}
\hline Name & Sense strand/sense primer (5'-3') & Antisense strand/antisense primer (5'-3') \\
\hline siRNA duplexes & & \\
FAM172A-siRNA1 & GAUAUGGAGUAAUAGUACUTT & AGUACUAUUACUCCAUAUCTT \\
FAM172A-siRNA2 & GAAGCGACGUGAUUUCUAUTT & AUAGAAAUCACGUCGCUUCTT \\
FAM172A-siRNA3 & CAAUCUAUGUUUGGGAUCATT & UGAUCCCAAACAUAGAUUGTT \\
FAM172A-siRNA4 & GACAGACUCUGUUCACAAUTT & AUUGUGAACAGAGUCUGUCTT \\
Control-siRNA & UUCUCCGAACGUGUCACGUTT & ACGUGACACGUUCGGAGAATT
\end{tabular}

Lentivirus construction and transfection. For transient overexpression and knockdown of miR-27a, micrOFF ${ }^{\mathrm{TM}}$ hsa-miR-27a inhibitor, micrOFF ${ }^{\mathrm{TM}}$ hsa-miR-27a mimic and micrON ${ }^{\mathrm{TM}}$ Negative Control (RiboBio, China) were at a final concentration of $50 \mathrm{nM}$ using Lipofectamine 3000 (Invitrogen). The efficacy of transfection was tested by real-time reverse transcription polymerase chain reaction (RT-PCR) analysis.

For FAM172A knockdown, three siRNAs (Sigma-Aldrich, USA) were designed against FAM172A (GenBank accession no. NM_032042.5). One control siRNA (Sigma-Aldrich) acted as a negative control, which exhibited no significant sequence similarity to human, mouse or rat gene sequence. On the basis of the manufacturer's instructions, transfection was performed with Lipofectamine 3000 (Invitrogen). The siRNA sequences are listed in Table II. For FAM172A overexpression, lentivirus was produced by transfecting HEK 293T packaging cells in DMEM (Hyclone) with a 3-plasmid system. DNA used for transfection was prepared by the mixture of pHelper 1.0, pHelper 2.0 and pLVX-IRES-Neo-FAM172A. The empty vector pLVX-IRES-Neo was purchased from Clontech Laboratories (Mountain View, CA, USA), and the pLVX-IRESNeo-FAM172A plasmid was generated by inserting FAM172A sequence. MDA-MB-231 cells were transduced with lentivirus in the presence of $6 \mu \mathrm{g} / \mathrm{ml}$ polybrene (Sigma-Aldrich) for $24 \mathrm{~h}$. Then cells were selected for 7 days in $2.5 \mathrm{mg} / \mathrm{ml}$ neomycin.

Cell proliferation assay. Cell Counting Kit-8 (CCK-8) (Dojindo) was used examining cell growth and viability. Cells were plated in 96-well plates at 10,000 cells/well in DMEM medium supplemented with $10 \%$ fetal bovine serum (FBS) and cultured at 24, 48, 72 and $96 \mathrm{~h}$ with 5 pmol miR-27a inhibitor, miR-con and miR-27a inhibitor + FAM172A siRNA respectively. The DMEM medium containing $10 \%$
CCK-8 was added to each well, which was a substitute for complete medium. Then the plates were incubated for $1 \mathrm{~h}$ in the incubator. Absorbance was measured at $490 \mathrm{~nm}$ using a Mithras LB 940 plate reader (Berthold, Germany).

Cell migration and invasion assay. The $8-\mu \mathrm{m}$ pore sized plain Transwell inserts (Costar, UK) for migration and the $8-\mu \mathrm{m}$ pore sized plain Transwell inserts with the BD Matrigel (BD Biosciences, USA) for invasion were placed in 24-well culture plates. Cells $\left(3 \times 10^{5}\right.$ per Transwell) for migration and cells $\left(1 \times 10^{5}\right.$ per Transwell) for invasion were seeded into the upper chamber, respectively, after suspended in serum-free DMEM. DMEM containing 5\% fetal bovine serum for migration and DMEM containing $10 \%$ fetal bovine serum for invasion served as a chemoattractant and were added to the bottom chamber of 24-well plates, respectively. After incubation of $48 \mathrm{~h}$, cells on the upper surface were removed. Cells that migrated to the lower surface were fixed and stained with $1 \%$ toluidine blue. Ten fields per experimental condition were randomly selected as previously described (32) and micrographed with IX71 microscope (Olympus, Japan) to quantify cells of migration and invasion. Images represent at least three independent experiments.

FAM172A rescue experiments. miR-27a inhibitor was cotransfected with FAM172A siRNA or the control siRNA. After transfection of $24 \mathrm{~h}$, cells were collected and used analyzing for proliferation, migration and invasion as described above. The expression of FAM172A was verified by western blotting.

Statistical analysis. The data are described as means \pm SEM from at least three independent experiments. The difference 
between two groups was analyzed using an independent Student's t-test. When comparing more than two groups, the difference was analyzed using one-way ANOVA analysis of variance. The difference between the expression level of miR-27a and the clinical variables was analyzed using Spearman correlation test. Receiver-operating characteristic (ROC) curves and the area under the ROC curve (AUC) were used for assessing the feasibility of miRNA as a diagnostic factor to detect CRC. The Kaplan-Meier analysis was used for analyzing the progression of CRC. Statistical analysis was performed using SPSS version 20.0 (SPSS, USA). P-values $<0.05$ were considered to indicate a statistical significance.

\section{Results}

miR-27a is upregulated in tissues of patients with CRC and CRC cell lines. Respectively, we determined miR-27a expression levels of six CRC cell lines (LoVo, HT-29, SW480, HCT116, DLD-1 and RKO) and a normal colorectal cell line (NCM460) which acts as a control. miR-27a expression levels in the six CRC lines were significantly higher than the NCM460 cells (6.12-, 3.56-, 5.48-, 3.96-, 4.37- and 3.58-fold, respectively) (Fig. 1A). We collected 60 pairs of CRC tissues and adjacent normal colorectal tissue (ANCT) to assess miR-27a expression. The expression levels of miR-27a in CRC tissues was higher than that of the ANCT samples (3.71-fold) $(3.92 \pm 0.50$ vs. $0.97 \pm 0.48, \mathrm{P}<0.001)$. On the contrary, the expression levels of FAM172A in CRC tissues was lower than that of the ANCT samples (3.03-fold) $(1.25 \pm 0.13$ vs. $0.38 \pm 0.11$, $\mathrm{P}<0.001$ ) (Fig. 1B).

miR-27a is a marker of diagnosis and prognosis in patients with colorectal cancer. First, the expression levels of miR-27a in tissues of 60 patients with CRC were evaluated by RT-qPCR. Also, we divided the patients into a high expression and a low expression group based on the median expression levels of miR-27a, which were used to analyse the group clinicopathological characteristics. We acquired data for age, sex, carcino-embryonic antigen (CEA), carbohydrate antigen 19-9 (CA19-9), TNM stage, distant metastasis, tumor size, and differentiation. Analysis showed that expression levels of miR-27a are closely related to the TNM stage $(\mathrm{P}=0.026)$ and distant metastasis $(\mathrm{P}=0.021)$ (Table III) which are all indicators of poor prognosis. Then we assigned patients to low-miR-27a group (expression $\mathrm{Ct}$ value $<22.50$ ) and high-miR-27a group (expression $\mathrm{Ct}$ value $\geq 22.50$ ) in order to determine correlation between the levels of miR-27a in CRC tissues and the survival of the CRC patients performed by a Kaplan-Meier survival analysis. The Kaplan-Meier analysis indicated that the low-miR-27a group had even better overall survival than the high-miR-27a group (Fig. 2A). These findings suggest that miR-27a is a useful biomarker of poor prognosis for patients with CRC. According to receiver-operating characteristic (ROC) curve analyses, miR-27a had the best sensitivity and specificity when the miR-27a $\mathrm{Ct}$ value was 22.50 (YI=0.633), and an AUC of $0.876(\mathrm{P}=0.0001 ; 95 \% \mathrm{CI}$, 0.787-0.964) (Fig. 2B) was obtained, which suggested that expression of miR-27a could discriminate between CRC and adjacent non-tumor colorectal tissues, thus may be used as a diagnostic marker for colorectal cancer.
Table III. Correlation between expression status of miR-27a and clinicopathological parameters.

\begin{tabular}{|c|c|c|c|c|}
\hline \multirow[b]{2}{*}{ Characteristics } & \multirow[b]{2}{*}{$\begin{array}{l}\text { No. of } \\
\text { patients }\end{array}$} & \multicolumn{2}{|c|}{$\begin{array}{c}\text { miR-27a } \\
\text { expression }\end{array}$} & \multirow[b]{2}{*}{ P-value } \\
\hline & & $\begin{array}{l}\text { Low, } \\
\mathrm{n}(\%)\end{array}$ & $\begin{array}{l}\text { High, } \\
\text { n (\%) }\end{array}$ & \\
\hline \multicolumn{5}{|c|}{ Patient age (years) } \\
\hline$\leq 60$ & 41 & $22(54)$ & $19(46)$ & 0.657 \\
\hline$>60$ & 19 & $9(47)$ & $10(53)$ & \\
\hline \multicolumn{5}{|l|}{ Sex } \\
\hline Female & 32 & $14(44)$ & $18(56)$ & 0.839 \\
\hline Male & 28 & $13(46)$ & $15(54)$ & \\
\hline \multicolumn{5}{|l|}{ CEA } \\
\hline Normal & 20 & $11(55)$ & $9(45)$ & 0.591 \\
\hline Elevated & 40 & $19(48)$ & $21(52)$ & \\
\hline \multicolumn{5}{|l|}{ CA19-9 } \\
\hline Normal & 18 & $8(45)$ & $10(52)$ & 0.825 \\
\hline Elevated & 42 & $20(48)$ & $22(52)$ & \\
\hline \multicolumn{5}{|l|}{ TNM stage } \\
\hline I and II & 27 & $15(56)$ & $12(44)$ & 0.026 \\
\hline III and IV & 33 & $9(27)$ & $24(73)$ & \\
\hline \multicolumn{5}{|c|}{ Distant metastasis } \\
\hline Absence & 36 & $24(67)$ & $12(33)$ & 0.021 \\
\hline Presence & 24 & $9(38)$ & $15(62)$ & \\
\hline
\end{tabular}

Tumor size

(cm)

$\begin{array}{lllll}\leq 4 & 26 & 12(46) & 14(54) & 0.610 \\ >4 & 34 & 18(53) & 16(47) & \end{array}$

Differentiation

$\begin{array}{lrrrr}\text { WD } & 18 & 8(44) & 10(56) & 0.315 \\ \text { MD } & 23 & 11(48) & 12(52) & \\ \text { PD } & 19 & 10(53) & 9(47) & \end{array}$

FAM172A

$\begin{array}{lrrrr}\geq 1 & 5 & 1(20) & 4(80) & <0.001 \\ <1 & 55 & 6(11) & 49(89) & \end{array}$

CEA, carcino-embryonic antigen; CA19-9, carbohydrate antigen 19-9; TNM, tumor node metastasis; WD, well differentiated; MD, moderately differentiated; PD, poorly differentiated. Bold print indicates a significant difference.

FAM172A is a direct target of $m i R-27 a$. In order to explore the regulation mechanism of miR-27a in CRC, we used bioinformatic analysis to predict target genes of miR-27a. As shown in Fig. 3A, there was a potential binding site of miR-27a predicted in the 3'-UTR of FAM172A. To test the specific regulation of this predicted binding site, we constructed a reporter vector which consisted of the luciferase coding sequence from the 
A

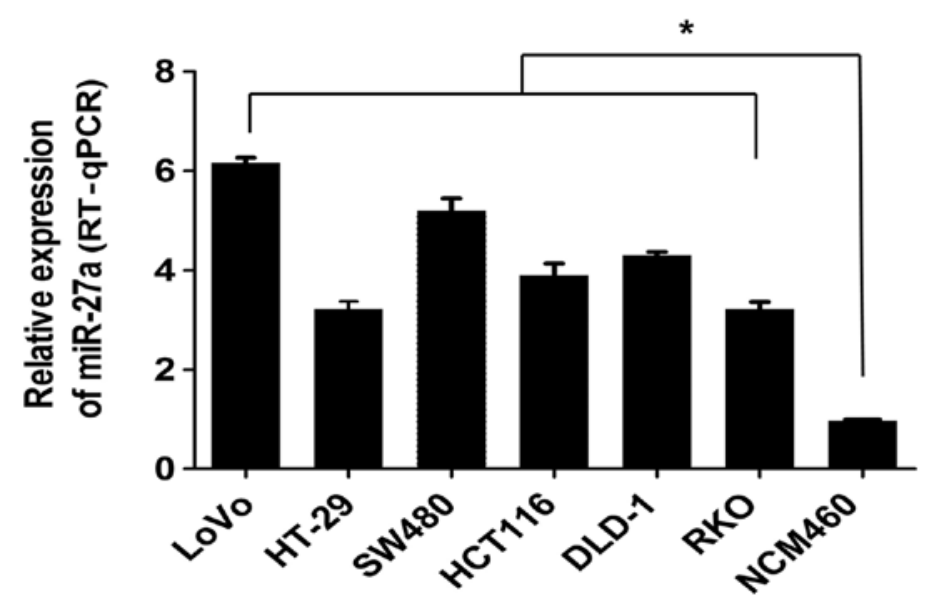

B
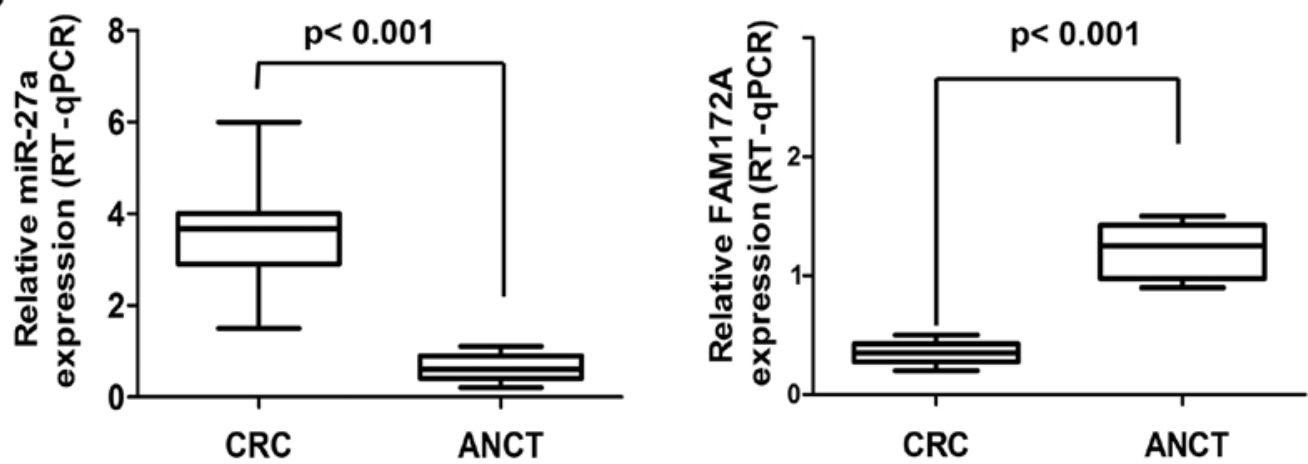

Figure 1. miR-27a is upregulated in CRC cell lines and tissues. (A) The expression of miR-27a expression was detected in colorectal cancer cell lines (LoVo, HT-29, SW480, HCT116, DLD-1 and RKO) and a normal human colonic epithelia cell line, NCM460. Data are shown as the mean \pm SD (n=3) in the cell lines, "P<0.05. (B) The expression levels of miR-27a (left) and FAM172A (right) in colorectal cancer ( $\mathrm{n}=60$ ) and adjacent normal mucosal tissues ( $\mathrm{n}=60)$ were determined by RT-qPCR analysis, ${ }^{*} \mathrm{P}<0.05$.

A

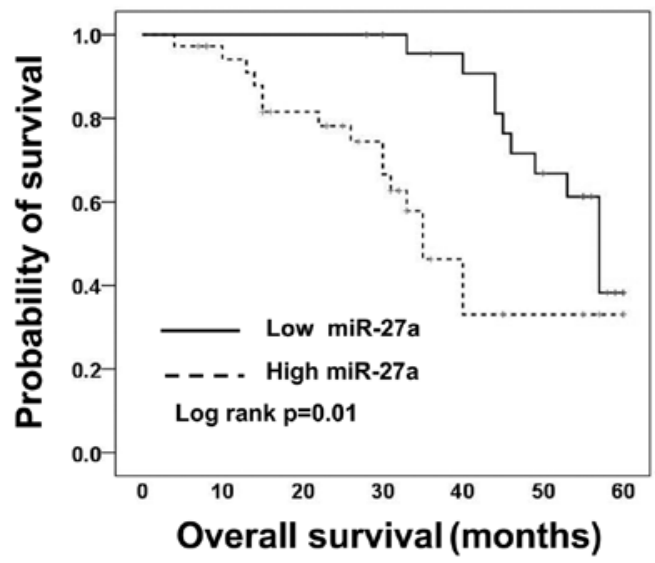

B

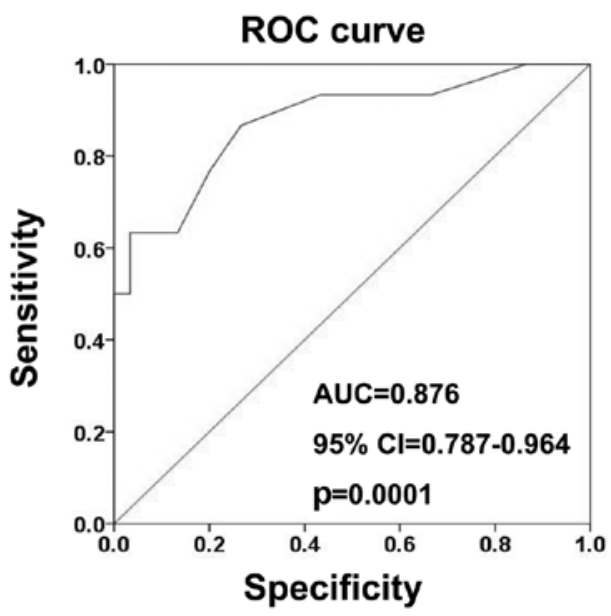

Figure 2. The roles of miR-27a in diagnosis and prognosis of colorectal cancer are shown. (A) Kaplan-Meier analysis for overall survival based on miR-27a expression. (B) ROC curve analysis showing performance of miR-27a expression to discriminate between colorectal cancer tissues and adjacent normal mucosal tissues. ROC, receiver-operating characteristic.

3'-UTR of FAM172A (Luc-FAM172A 3'-UTR). The mutant was prepared which consisted of the putative binding sites (Luc-FAM172A-mut 3'-UTR) (Fig. 3A). Cotransfection experiments indicated that miR-27a decreased the luciferase activity of Luc-FAM172A 3'-UTR but had very little influence on Luc-FAM172A-mut 3'-UTR (Fig. 3B), which demonstrated that FAM172A is a potential target of miR-27a. miR-27a was downregulated or upregulated in CRC cell lines transfected with the miR-27a inhibitor or mimic, respectively compared with the control-miR group (Fig. 3C). 
A

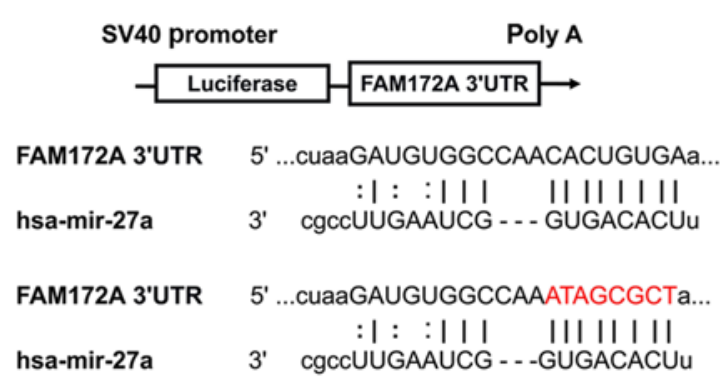

C

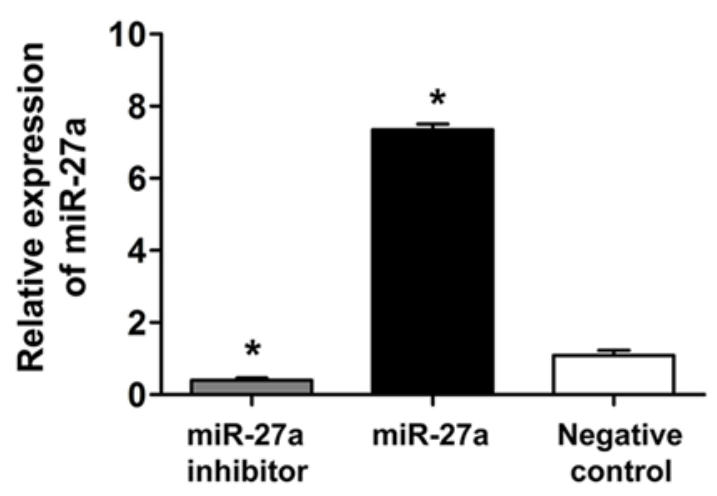

LoVo
B
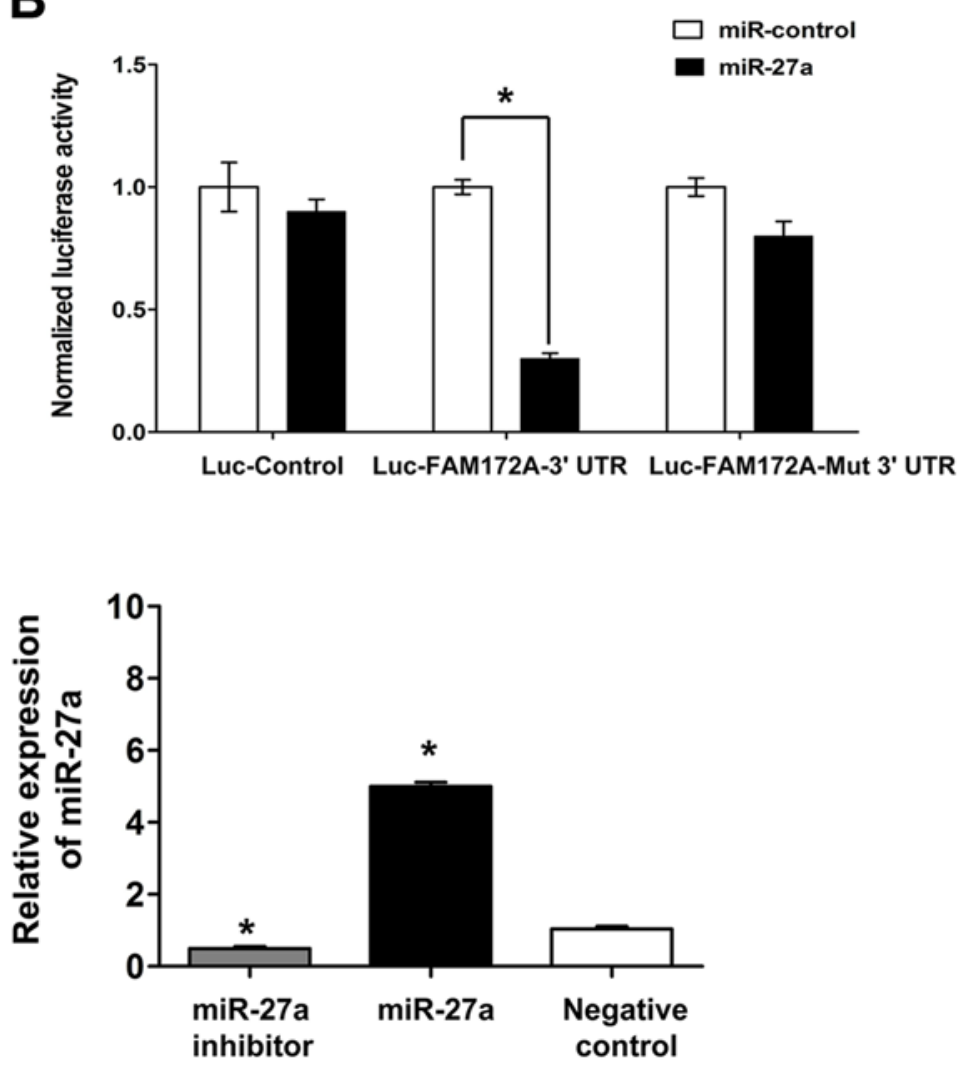

SW480

Figure 3. miR-27a directly targets FAM172A. (A) The predicted binding sequences of miR-27a in the 3'-UTR of FAM172A. (B) Luciferase reporter assay found that the HEK293T cells co-transfected with miR-27a mimics and wild-type (WT) FAM172A 3'-UTR caused a significant decrease in luciferase activity, whereas co-transfection with mutant-type (MUT) FAM172A 3'-UTR and miR-27a mimics showed no significant difference with the control group cells co-transfected with untreated psiCHECK vector (vector) and a Renilla luciferase vector. Firefly luciferase activity was used normalizing Renilla luciferase activity, ${ }^{*} \mathrm{P}<0.05$. (C) miR-27a expression was detected in the LoVo and SW480 colorectal cell lines after transfecting miR-27a mimic, miR-27a inhibitor and cont-miR, ${ }^{*} \mathrm{P}<0.05$.

MicroRNA-27a plays a negative regulation role in FAM172A expression. In order to determine whether miR-27a is a regulator in FAM172A expression, we utilized LoVo and SW480 cells transfected with control-miR, miR-27a mimic or miR-27a inhibitor, respectively. Suppression of miR-27a in colorectal cancer cells resulted in a 4- to 6-fold increase in FAM172A mRNA levels and overexpression of miR-27a led to a 40-60\% reduced FAM172A mRNA levels (Fig. 4A). As shown in Fig. 4B, an approximately 2- to 3 -fold increase in protein levels of FAM172A in colorectal cancer cells transfected with inhibition of miR-27a, and overexpression of miR-27a resulted in $\sim 30-40 \%$ reduced protein levels in both LoVo and SW480 cells.

miR-27a inhibitor decreases proliferation, migration and invasion of colon cancer cells, which could be partially rescued by decreased FAM172A. The role of miR-27a in the regulation of proliferation, migration and invasion of CRC cells was further investigated. We transfected the LoVo and SW480 CRC cell lines with miR-27a inhibitor. As shown in Fig. 5A, we found that the cells transfected with the miR-27a inhibitor obviously decreased proliferation compared with the cells transfected with control-miR at 48,72 and $96 \mathrm{~h}$ by cell proliferation assay, but there was no obvious difference in proliferation between CRC cells transfected with miR-27a inhibitor and the control at $24 \mathrm{~h}$. Compared with the negative control, both the migration and invasion of CRC cells transfected with miR-27a inhibitor were reduced at $48 \mathrm{~h}$ by Transwell migration and Matrigel invasion assays (Fig. 5B and C).

Moreover, we tested the levels of $N F-\kappa B, M M P-2, M M P-9$ and FAM172A mRNA and proteinin LoVo and SW480 cells transfected with miR-27a inhibitor and the negative control respectively. The results indicated that the mRNA and protein levels of these genes were decreased in CRC cell lines transfected with miR-27a inhibitor, as compared with the negative control (Fig. 6). In order to prove whether FAM172A mediates the effects of miR-27a on migration and invasion, we performed cell assays in LoVo and SW480 cell lines co-transfected with miR-27a inhibitor and FAM172A-siRNA. As shown in Fig. 5, the results suggested that FAM172A-siRNA to some degree rescued the proliferation, migration and invasion in LoVo and SW480 cell lines transfected with miR-27a inhibitor, and decreased expression of FAM172A rescued the mRNA and protein expression levels of NF- $\kappa \mathrm{B}, \mathrm{MMP}-2, \mathrm{MMP}-9$ and FAM172A (Fig. 6). 
A

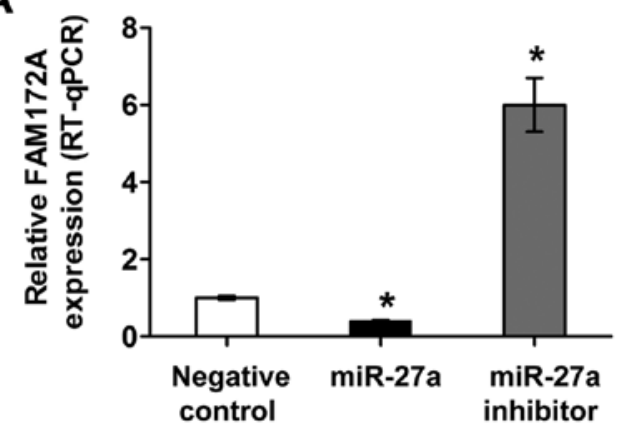

B

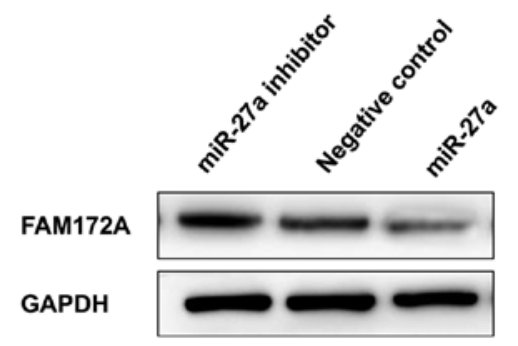

C

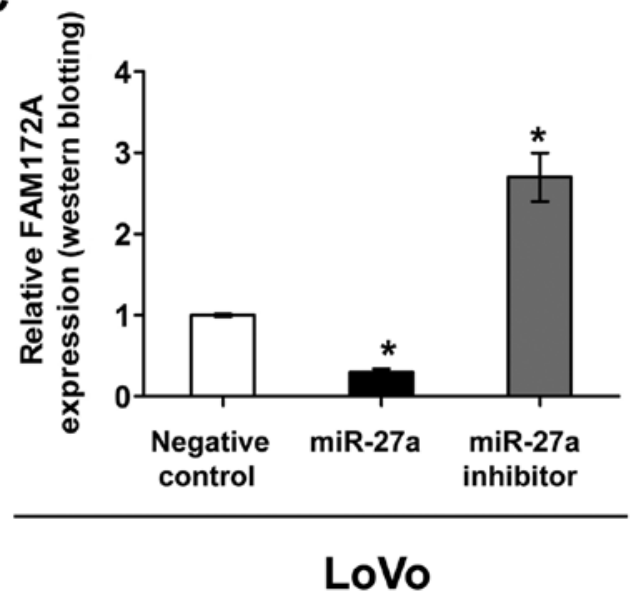

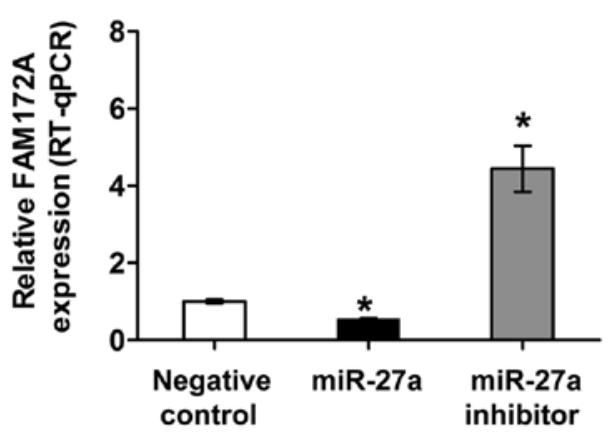

GAPDH
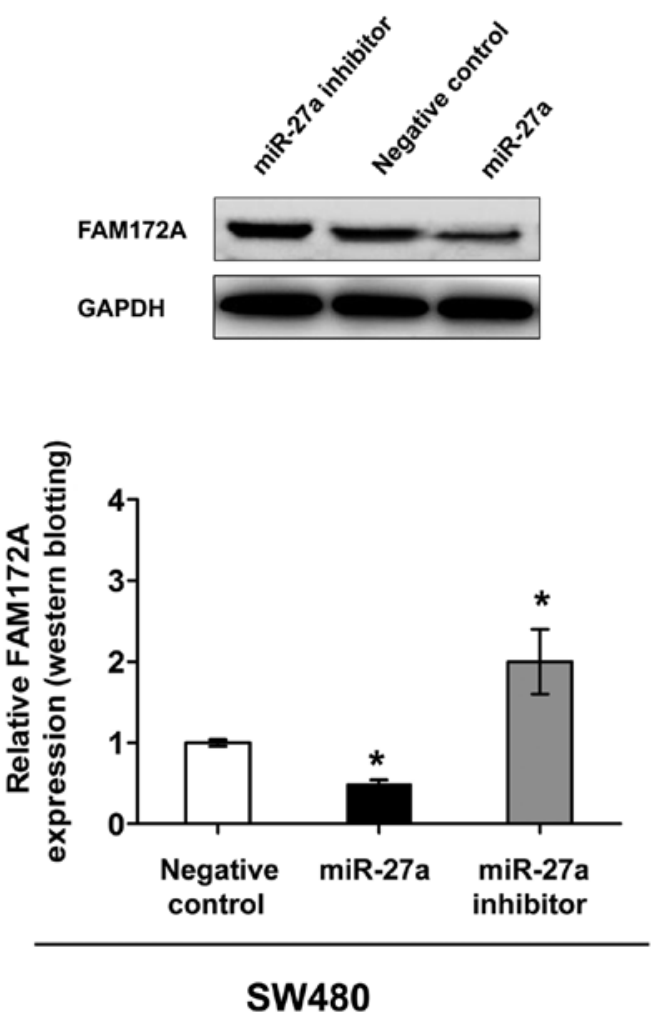

Figure 4. miR-27a negativly regulated the FAM172A expression in CRC cell lines. (A,B) The mRNA levels of FAM172A expression was examined by RT-qPCR in the LoVo and SW480 colorectal cell lines following transfection with miR-27a mimic, miR-27a inhibitor and cont-miR, ${ }^{*} \mathrm{P}<0.05$. (C) The protein levels of FAM172A expression was examined by western blotting in the LoVo and SW480 colorectal cell lines following transfection with miR-27a mimic, miR-27a inhibitor and cont-miR, ${ }^{*} \mathrm{P}<0.05$.

\section{Discussion}

As a protein of 416 amino acid, FAM172A belongs to the UPF0528 family and its protein-coding gene is located in human chromosome 5 (9). Our previous research showed that FAM172A expression had a lower expression in CRC tissues of patients with metastasis compared with CRC tissues of patients without metastasis (11). However, relevant studies of FAM172A and its more unknown functions in CRC are still scarce.

There is increasing evidence that dysregulation of miRNAs is involved in colorectal cancer. They bind to the 3'-untranslated region (UTR) of target genes, which promotes RNA degradation and even regulate mRNA transcription (33), thus regulating the biological function of cancer cells, including cell proliferation, migration and invasion. Interestingly, our previous research showed that STAT1 affected the expression of FAM172A via binding to the FAM172A promoter. After co-transfection of STAT1 overexpression plasmid or siRNA in LoVo cells, the transcriptional activity of FAM172A was powerfully increased (12). Therefore, FAM172A expression in colorectal cancer cells may depend on its transcriptional regulation. Our present study found that the miR-27a seed sequence has a complementarity to the 3 '-UTR nucleotide sequence of human FAM172A mRNA. miR-27a has been closely related to the tumor process, and it has been shown to regulate the biological function of cancer cells, including cell proliferation, migration and invasion by affecting target genes $(30,31)$. We provided evidence that miR-27a decreased the luciferase energy of Luc-FAM172A 3'-UTR but barely had an effect on Luc-FAM172A-mut 3'-UTR, indicating that FAM172A was a direct target of miR-27a by luciferase 
A

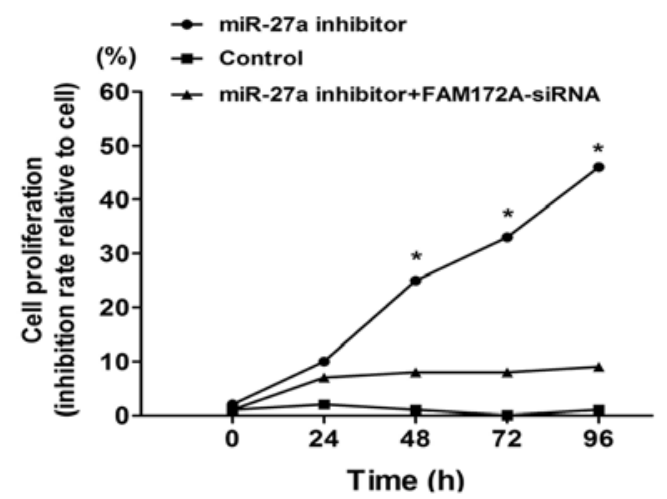

B

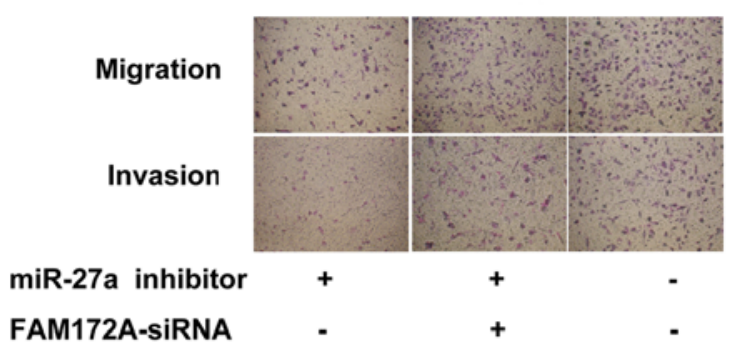

C
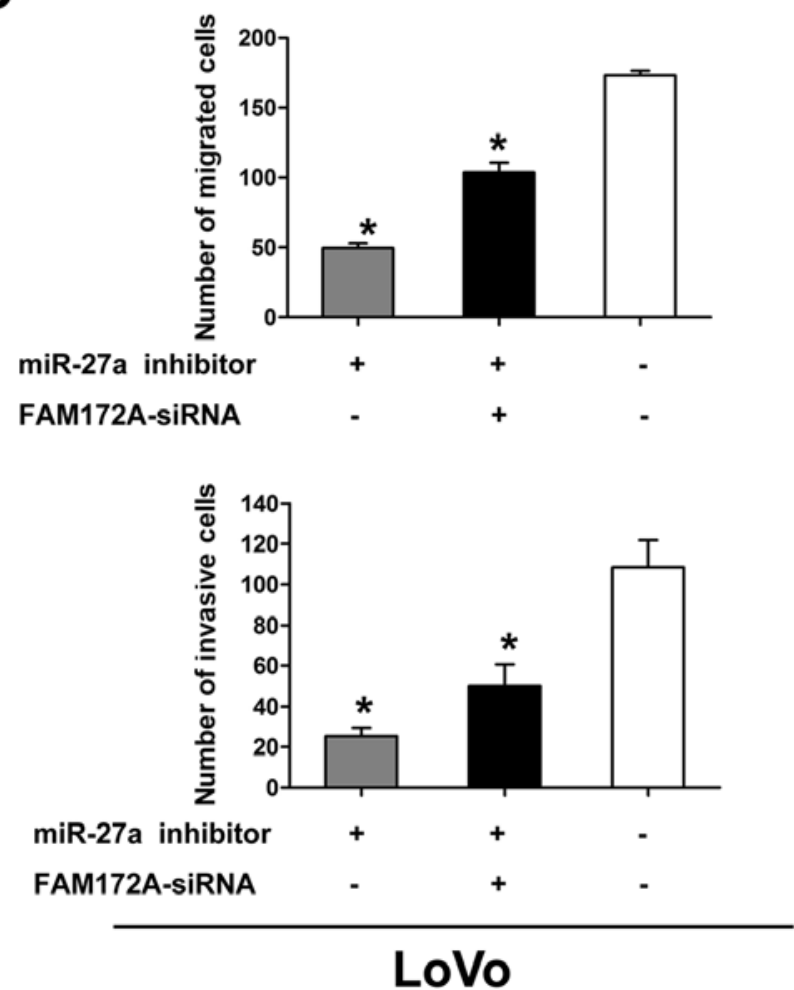

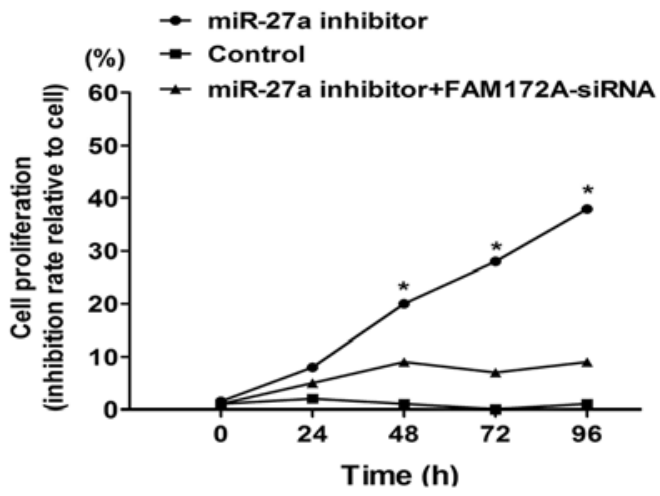

Migration

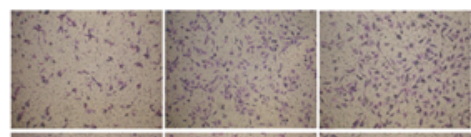

Invasion

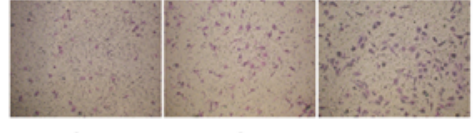

miR-27a inhibitor

FAM172A-siRNA
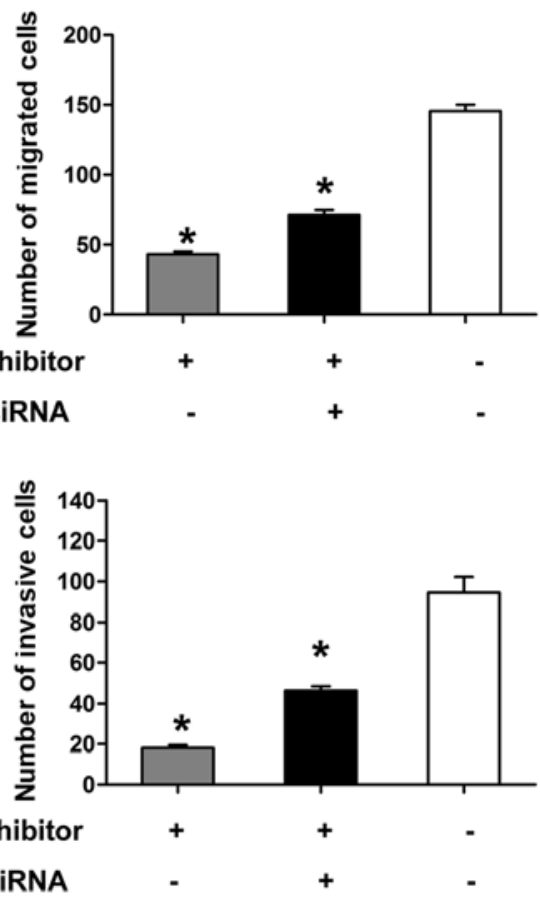

SW480

Figure 5. miR-27a inhibitor-induced suppression of proliferation, migration and invasion of CRC cell lines were rescued by siRNA of FAM172A. (A) CCK-8 assays were conducted on CRC cells transfected with miR-27a inhibitor $(50 \mathrm{nmol} / \mathrm{l})$, miR-27a inhibitor + FAM172A-siRNA or control-miR, respectively. The cell lines transfected with miR-27a inhibitor demonstrated significantly the decreased ability of proliferation as compared to control lines at 48,72 and $96 \mathrm{~h}$. FAM172A-siRNA reversed the effect of miR-27a inhibitor on cells, ${ }^{*} \mathrm{P}<0.05$. (B) Representative images depicting cell migration and invasion assays, ${ }^{\text {}} \mathrm{P}<0.05$. (C) For each cell line, miR-27a inhibitor resulted in decreased migration and invasion as compared to the control. FAM172A siRNA reversed the effect of miR-27a inhibitor on cell migration in both cell lines, ${ }^{*} \mathrm{P}<0.05$

reporter assay. Furthermore, both mRNA and protein levels of FAM172A was downregulated in LoVo and SW480 cells transfected with miR-27a. The silencing of miR-27a can upregulate the mRNA level and protein levels of FAM172A, 
A

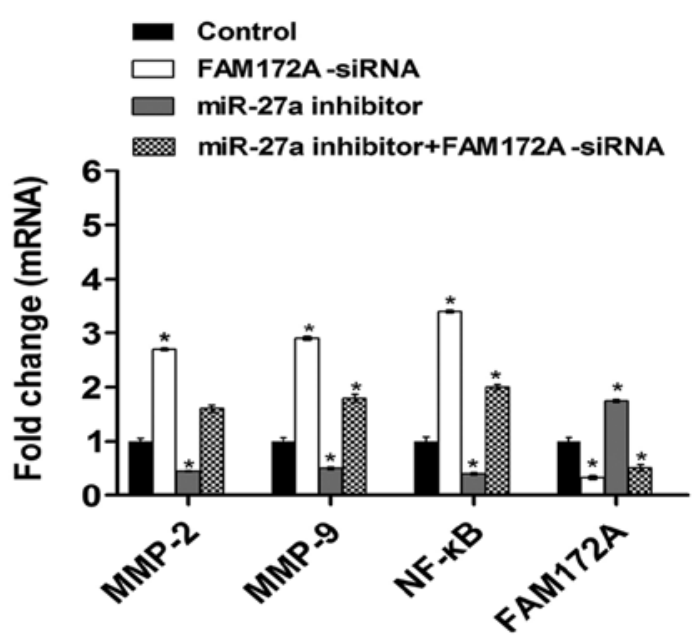

B

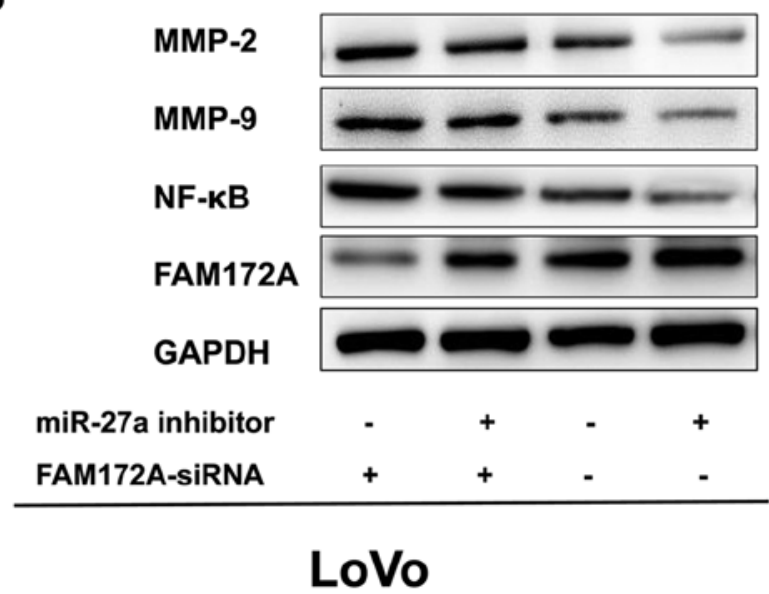

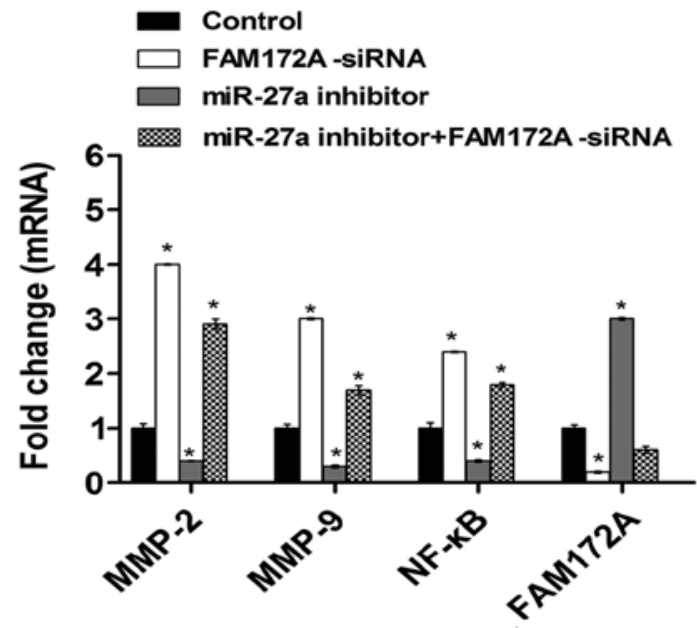

MMP-2

MMP-9

NF-KB

FAM172A

GAPDH
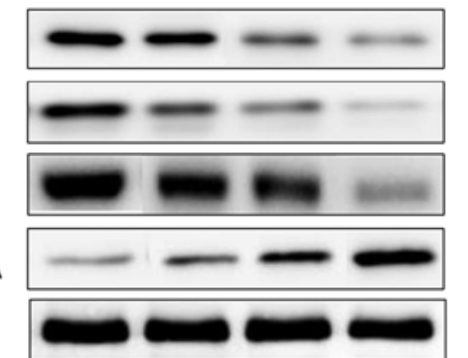

miR-27a inhibitor

FAM172A-siRNA

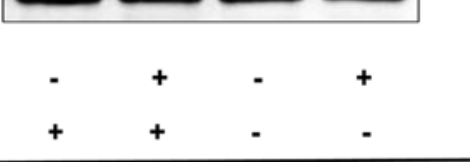

SW480

Figure 6. miR-27a inhibitor suppresses levels of NF- $\kappa$ B, MMP-2, MMP-9 and FAM172A expression. (A) CRC lines were transfected with miR-27a inhibitor ( $50 \mathrm{nmol} / 1)$, miR-27a inhibitor + FAM172A-siRNA or control, followed by RT-qPCR analysis of the NF- $\kappa$ B, MMP-2, MMP-9 and FAM172A mRNA, "P<0.05. (B) CRC lines were transfected with miR-27a inhibitor (50 nmol/1), miR-27a inhibitor + FAM172A-siRNA or control, followed by western blot analysis of the NF- $\mathrm{B}, \mathrm{MMP}-2, \mathrm{MMP}-9$ and FAM172A protein, ${ }^{*} \mathrm{P}<0.05$.

indicating that miR-27a participates in regulating FAM172A expression. Therefore FAM172A is a downstream target gene of miR-27a.

Our prior studies showed that overexpression of FAM172A inhibited the migrational capability and cell invasiveness of CRC cell lines by suppressing the expression of MMPs (11). To further explore the biological functions of FAM172A regulated by miR-27a in LoVo and SW480 cell lines, we determine the effect of miR-27a on cell poliferation, migration and invasion. In this study, we first confirmed that cell migration and invasion become weaken in CRC cells transfected with miR-27a inhibitor, which can be significantly rescued by FAM172A siRNA. Interestingly, mRNA and protein levels of MMP-2, MMP-9 and FAM172A are decreased in CRC cells transfected with miR-27a inhibitor, which also can be significantly rescued by FAM172A siRNA. Accordingly, miR-27a-FAM172A axis is linked to the migration and invasion of LoVo and SW480 cell lines. Cell migration and invasion occur in the tumorigenic process. Therefore, migration and invasion can resulted in metastasis, thus causing deaths of $\sim 90 \%$ of cancer patients (34). MMPs are critical players in tumor migration and invasion through degradation of ECM components. Other studies have shown that MMP-2 expression is significantly relevant for the tumor metastasis (35) and expression of MMP-9 is closely related to poor prognosis and metastasis of CRC (36). MMP-2 and MMP-9 are frequently upregulated in cancer cells $(37,38)$. Thus miR-27a-mediated novel targets contribute to the metastatic pathways.

Our previous results manifested that upregulation of FAM172A inhibited cell proliferation in LoVo and SW480 cell lines. Instead, proliferation of these cells treated with FAM172A siRNA was induced significantly (12). In the present study, we found that FAM172A overexpression decreased the expression of $\mathrm{NF}-\kappa \mathrm{B}$. In addition, significantly decreased proliferation and expression of $N F-\kappa B$ are observed in the miR-27a inhibition group, which can be significantly rescued by FAM172A siRNA. Accordingly, miR-27a-FAM172A axis is linked to the proliferation of CRC cells. Some research has shown that NF- $\kappa \mathrm{B}$ are also related to cell migration and invasion $(39,40)$. The NF- $\mathrm{B}$ signaling pathway has some effect on human health and disease, particularly in cancers. Activation of $N F-\kappa B$ is frequently involved in a variety of tumors, thus promoting the development of tumors, which may lead to different etiology and pathogenesis, like mutations or deletions 
of I $\kappa \mathrm{B} \alpha$ in Hodgkin's lymphoma, amplifications or translocations of NIK in multiple myeloma $(41,42)$. Deregulation of the miRNAs also leads to the abnormal activation of the $\mathrm{NF}-\kappa \mathrm{B}$ pathway by regulating the $\mathrm{NF}-\kappa \mathrm{B}$ pathway except the genomic aberrations of protein-coding genes. Thus, a deeper understanding of $\mathrm{NF}-\kappa \mathrm{B}$ signaling may contribute to progress for determination of the molecular pathology of a variety of cancer. miRNAs may provide novel targets for anticancer therapy by regulating the $\mathrm{NF}-\kappa \mathrm{B}$ activity.

In conclusion, higher expression of miR-27a was observed in CRC tissues compared with ANCT. There was a positive correlation between the expression of miR-27a and some clinicopathological characteristics and overall survival of patients with CRC. We proposed a novel regulatory mechanism miR-27a-FAM172A axis for cell proliferation, migration and invasion in CRC based on our observations. miR-27a is an oncogenic miRNA and a direct regulator of FAM172A in CRC. The identification of this novel molecular pathway mediated by the miR-27a-FAM172A axis and its role may lead to a deeper knowledge on CRC tumorigenesis, thus providing guiding reference for future research and patient stratification according to miR-27a/FAM172A expression levels, which ultimately designs novel individual treatments.

\section{Acknowledgements}

This study was supported by the Guangdong Natural Science Foundation (2014A030313324) and the Guangdong Natural Science Foundation (2015) and the Key Clinical Specialty Discipline Construction Program and Undergraduate Training Program for Innovation and Entrepreneurship (201512121116).

\section{References}

1. Jemal A, Siegel R, Xu J and Ward E: Cancer statistics, 2010. CA Cancer J Clin 60: 277-300, 2010.

2. Steeg PS: Metastasis suppressors alter the signal transduction of cancer cells. Nat Rev Cancer 3: 55-63, 2003.

3. Peltomäki P: Role of DNA mismatch repair defects in the pathogenesis of human cancer. J Clin Oncol 21: 1174-1179, 2003.

4. Yun J, Rago C, Cheong I, Pagliarini R, Angenendt P, Rajagopalan H, Schmidt K, Willson JK, Markowitz S, Zhou S, et al: Glucose deprivation contributes to the development of KRAS pathway mutations in tumor cells. Science 325: 1555-1559, 2009.

5. Kim TM, Laird PW and Park PJ: The landscape of microsatellite instability in colorectal and endometrial cancer genomes. Cell 155: 858-868, 2013.

6. Bostick M, Kim JK, Estève PO, Clark A, Pradhan S and Jacobsen SE: UHRF1 plays a role in maintaining DNA methylation in mammalian cells. Science 317: 1760-1764, 2007.

7. Fidler IJ: Critical determinants of metastasis. Semin Cancer Biol 12: 89-96, 2002.

8. Li L, Dong X, Leong MC, Zhou W, Yang Z, Chen F, Bao Y, Jia W and $\mathrm{Hu}$ R: Identification of the novel protein FAM172A, and its up-regulation by high glucose in human aortic smooth muscle cells. Int J Mol Med 26: 483-490, 2010.

9. Li LX, Tao Z, Dong XH, Liang WC, Yang ZH, Mou B, Bao YQ, Wang C, Jia WP and Hu RM: Molecular cloning of a novel gene, C5orf21 gene and its roles in diabetic macroangiopathy. Zhonghua Yi Xue Za Zhi 89: 2574-2577, 2009 (In Chinese).

10. Cui C, Ye L, Huang Z, Huang S, Liu H and Yu J: FAM172A is a tumor suppressor in colorectal carcinoma. Tumour Biol 37: 6501-6510, 2016.

11. Qian K, Zhang J, Lu J, Liu W, Yao X, Chen Q, Lu S, Xiang G and Liu H: FAM172A modulates apoptosis and proliferation of colon cancer cells via STAT1 binding to its promoter. Oncol Rep 35: 1273-1280, 2016.
12. Babashah $\mathrm{S}$ and Soleimani $\mathrm{M}$ : The oncogenic and tumour suppressive roles of microRNAs in cancer and apoptosis. Eur $\mathrm{J}$ Cancer 47: 1127-1137, 2011.

13. Calin GA and Croce CM: MicroRNA signatures in human cancers. Nat Rev Cancer 6: 857-866, 2006.

14. Ventura A and Jacks T: MicroRNAs and cancer: Short RNAs go a long way. Cell 136: 586-591, 2009.

15. Bartel DP: MicroRNAs: Genomics, biogenesis, mechanism, and function. Cell 116: 281-297, 2004.

16. Filipowicz W, Bhattacharyya SN and Sonenberg N: Mechanisms of post-transcriptional regulation by microRNAs: Are the answers in sight? Nat Rev Genet 9: 102-114, 2008.

17. Iorio MV and Croce CM: MicroRNAs in cancer: Small molecules with a huge impact. J Clin Oncol 27: 5848-5856, 2009.

18. Place RF, Li LC, Pookot D, Noonan EJ and Dahiya R: MicroRNA-373 induces expression of genes with complementary promoter sequences. Proc Natl Acad Sci USA 105: 1608-1613, 2008.

19. Hobert O: Gene regulation by transcription factors and microRNAs. Science 319: 1785-1786, 2008.

20. Garzon R, Calin GA and Croce CM: MicroRNAs in cancer. Annu Rev Med 60: 167-179, 2009.

21. Lim LP, Lau NC, Garrett-Engele P, Grimson A, Schelter JM, Castle J, Bartel DP, Linsley PS and Johnson JM: Microarray analysis shows that some microRNAs downregulate large numbers of target mRNAs. Nature 433: 769-773, 2005.

22. Liu M and Chen H: The role of microRNAs in colorectal cancer. J Genet Genomics 37: 347-358, 2010.

23. Michael MZ, O' Connor SM, van Holst Pellekaan NG, Young GP and James RJ: Reduced accumulation of specific microRNAs in colorectal neoplasia. Mol Cancer Res 1: 882-891, 2003.

24. Volinia S, Calin GA, Liu CG, Ambs S, Cimmino A, Petrocca F, Visone R, Iorio M, Roldo C, Ferracin M, et al: A microRNA expression signature of human solid tumors defines cancer gene targets. Proc Natl Acad Sci USA 103: 2257-2261, 2006.

25. Cekaite L, Rantala JK, Bruun J, Guriby M, Agesen TH, Danielsen SA, Lind GE, Nesbakken A, Kallioniemi O, Lothe RA, et al: MiR-9, -31 , and -182 deregulation promote proliferation and tumor cell survival in colon cancer. Neoplasia 14: 868-879, 2012.

26. Valeri N, Braconi C, Gasparini P, Murgia C, Lampis A, Paulus-Hock V, Hart JR, Ueno L, Grivennikov SI, Lovat F, et al: MicroRNA-135b promotes cancer progression by acting as a downstream effector of oncogenic pathways in colon cancer. Cancer Cell 25: 469-483, 2014.

27. Rokavec M, Öner MG, Li H, Jackstadt R, Jiang L, Lodygin D, Kaller M, Horst D, Ziegler PK, Schwitalla S, et al: IL-6R/STAT3/ miR-34a feedback loop promotes EMT-mediated colorectal cancer invasion and metastasis. J Clin Invest 124: 1853-1867, 2014.

28. Wang F, Yang YZ, Shi CZ, Zhang P, Moyer MP, Zhang HZ, Zou Y and Qin HL: UHRF1 promotes cell growth and metastasis through repression of p16(ink4a) in colorectal cancer. Ann Surg Oncol 19: 2753-2762, 2012.

29. Colangelo T, Polcaro G, Ziccardi P, Pucci B, Muccillo L, Galgani M, Fucci A, Milone MR, Budillon A, Santopaolo M, et al: Proteomic screening identifies calreticulin as a miR-27a direct target repressing MHC class I cell surface exposure in colorectal cancer. Cell Death Dis 7: e2120, 2016.

30. Zhou L, Liang X, Zhang L, Yang L, Nagao N, Wu H, Liu C, Lin S, Cai G and Liu J: MiR-27a-3p functions as an oncogene in gastric cancer by targeting BTG2. Oncotarget 7: 51943-51954, 2016.

31. Livak KJ and Schmittgen TD: Analysis of relative gene expression data using real-time quantitative PCR and the 2(-Delta Delta C(T)) method. Methods 25: 402-408, 2001.

32. De Bacco F, Luraghi P, Medico E, Reato G, Girolami F, Perera T, Gabriele P, Comoglio PM and Boccaccio C: Induction of MET by ionizing radiation and its role in radioresistance and invasive growth of cancer. J Natl Cancer Inst 103: 645-661, 2011.

33. Spizzo R, Nicoloso MS, Croce CM and Calin GA: SnapShot: MicroRNAs in cancer. Cell 137: 586-586.e1, 2009.

34. Lee HJ, Song IC, Yun HJ, Jo DY and Kim S: CXC chemokines and chemokine receptors in gastric cancer: From basic findings towards therapeutic targeting. World J Gastroenterol 20: 1681-1693, 2014.

35. Shen W, Xi H, Wei B and Chen L: The prognostic role of matrix metalloproteinase 2 in gastric cancer: A systematic review with meta-analysis. J Cancer Res Clin Oncol 140: 1003-1009, 2014. 
36. Liu Y, Liu H, Luo X, Deng J, Pan Y and Liang H: Overexpression of SMYD3 and matrix metalloproteinase-9 are associated with poor prognosis of patients with gastric cancer. Tumour Biol 36: 4377-4386, 2015.

37. Adiseshaiah P, Vaz M, Machireddy N, Kalvakolanu DV and Reddy SP: A Fra-1-dependent, matrix metalloproteinase driven EGFR activation promotes human lung epithelial cell motility and invasion. J Cell Physiol 216: 405-412, 2008.

38. Belguise K, Kersual N, Galtier F and Chalbos D: FRA-1 expression level regulates proliferation and invasiveness of breast cancer cells. Oncogene 24: 1434-1444, 2005.
39. Li N, Fu H, Tie Y, Hu Z, Kong W, Wu Y and Zheng X: miR-34a inhibits migration and invasion by down-regulation of c-Met expression in human hepatocellular carcinoma cells. Cancer Lett 275: 44-53, 2009.

40. Westermarck J and Kähäri VM: Regulation of matrix metalloproteinase expression in tumor invasion. FASEB J 13: 781-792, 1999.

41. Staudt LM: Oncogenic activation of NF-kappaB. Cold Spring Harb Perspect Biol 2: a000109, 2010.

42. Ben-Neriah Y and Karin M: Inflammation meets cancer, with $\mathrm{NF}-\kappa \mathrm{B}$ as the matchmaker. Nat Immunol 12: 715-723, 2011. 